\title{
Enseigner la géographie à l'école primaire et la genèse des identités territoriales en Europe au tournant du XVIII ${ }^{\mathrm{e}}$ au XIX ${ }^{\mathrm{e}}$ siècle
}

\section{Nicola Todorov}

\section{OpenEdition}

\section{Journals}

Édition électronique

URL : https://journals.openedition.org/geohist/1301

DOI : 10.4000/geohist.1301

ISSN : 2264-2617

Éditeur

Association française de la Revue de géographie historique

\section{Référence électronique}

Nicola Todorov, «Enseigner la géographie à l'école primaire et la genèse des identités territoriales en Europe au tournant du XVIII au XIXe siècle », Revue de géographie historique [En ligne], 13 | 2018, mis en ligne le 20 novembre 2018, consulté le 12 juin 2021. URL : http://journals.openedition.org/geohist/ 1301 ; DOI : https://doi.org/10.4000/geohist.1301

Ce document a été généré automatiquement le 12 juin 2021.

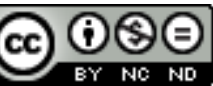

Ce(tte) œuvre est mise à disposition selon les termes de la Licence Creative Commons Attribution -

Pas d'Utilisation Commerciale - Pas de Modification 4.0 International. 


\title{
Enseigner la géographie à l'école primaire et la genèse des identités territoriales en Europe au tournant du XVIII ${ }^{\mathrm{e}}$ au XIX ${ }^{\mathrm{e}}$ siècle
}

\author{
Nicola Todorov
}

Le rôle de l'enseignement de la géographie dans le développement du sentiment national et dans la genèse des identités territoriales a été reconnu de longue date et a fait l'objet d'études approfondies notamment pour l'école de la Troisième République dans un contexte de défaite contre la Prusse en 1870 (Lefort, 1998), de même que l'on a souligné toute l'ambiguïté du concept d'identité territoriale (Guermond, 2006). En revanche, l'idée d'une fonction déterminante occupée par la période révolutionnaire et napoléonienne dans l'émergence des nationalismes européens et d'identités territoriales liées à l'État-nation, communément admise jusqu'aux années 1950, a été fortement mise en doute depuis quelques décennies et divise les spécialistes. Loin de correspondre à un éveil des masses populaires, mobilisées dans toute l'Europe déchirée par une vingtaine d'années de guerre, la période révolutionnaire et napoléonienne n'aurait pas fait naître le sentiment national, notamment dans les pays tels que l'Italie ou l'Allemagne. Celui-ci n'aurait préoccupé que les élites instruites (Planert, 2016, Jeismann, 1992). Même pour la France, certains auteurs mettent en doute qu'au début $\mathrm{du} \mathrm{XIX}^{\mathrm{e}}$ siècle, le cadre national ait correspondu au territoire identitaire principal des petites gens (Marzagalli, 2016). Pour que l'État-nation devienne l'affaire des «masses ", il aurait fallu à la fois les transformations de l'industrialisation, conduisant à une augmentation notable de la proportion des couches sociales instruites, et la diffusion massive de l'instruction primaire et secondaire. Le constat d'un éclatement de la géographie savante, passant de l'universalisme des Lumières à l'émergence d'écoles de géographie nationales (Péaud, 2014), ne contredit pas cette interprétation dans la mesure où la géographie savante intéressait une partie des élites intellectuelles et qu'un décalage temporel entre les productions scientifiques et leur large réception n'aurait rien de surprenant. Dans le cas de l'enseignement primaire, on a cependant 
postulé il y a quelques décennies que les invasions françaises d'une bonne partie de l'Europe au tournant du XVIII ${ }^{e}$ au XIX ${ }^{e}$ siècle auraient incité les États à initier une politique d'éducation populaire afin de mieux mobiliser les masses contre cette «menace » (Boli, Ramirez, Meyer,1985). Certaines recherches récentes tentent de déceler les conséquences lointaines de cette impulsion donnée au début du XIX ${ }^{\mathrm{e}}$ siècle (Korthals, 2016). Il convient cependant de souligner que l'historiographie a depuis longtemps identifié d'autres acteurs promoteurs de l'enseignement primaire que les autorités étatiques (Neugebauer, 1985).

2 Alors, si l'école devait éduquer les couches sociales inférieures conformément aux besoins des États, il est légitime de s'interroger quel rôle fut dévolu à l'enseignement de la géographie à l'époque révolutionnaire et napoléonienne, à l'instar de ce qui se pratiquera des décennies plus tard. Cet article se propose alors d'étudier l'histoire de l'enseignement de la géographie dans les écoles primaires pendant la période révolutionnaire au sens large et en même temps d'en reconstituer, ne serait-ce que d'une façon panoramique et lacunaire, la géographie de cet enseignement dans cette Europe bouleversée par la Révolution.

\section{L'école primaire à la fin du XVIIle siècle - des situations et des enjeux différents}

\section{A. Des écarts d'alphabétisation et de densité des réseaux scolaires}

3 Au tournant du XVIII ${ }^{e}$ au XIX ${ }^{e}$ siècle, les situations des écoles élémentaires ou primaires sont extrêmement différentes d'un bout à l'autre de l'Europe. L'une des premières fonctions de l'école primaire est d'enseigner la lecture et l'écriture. Même si elles ne sont établies quantitativement avec une représentativité suffisante que pour le milieu du XIX ${ }^{e}$ siècle, les inégalités de l'alphabétisation sont un indicateur de la densité du réseau scolaire élémentaire. Grossièrement, une Europe du Sud, moins alphabétisée, s'oppose à une Europe du Nord-Ouest et du Nord, où les taux d'alphabétisation sont plus élevés, alors que vers l'Europe centrale et orientale les taux d'alphabétisation déclinent.

4 Par exemple, lorsqu'en 1795/1796, l'administration de la province de Prusse méridionale composée de territoires annexés lors des derniers partages de la Pologne, à peine $14 \%$ des maires nommés savaient lire et écrire dans 1708 villages pour lesquels nous disposons d'informations relativement précises ${ }^{1}$. Dans le futur duché de Varsovie (1807-1813), dans trois districts pour lesquels nous disposons de données, les taux d'alphabétisation des conseillers municipaux, qui sont issus de couches sociales plus larges que le groupe des maires, allaient de 0 à moins de 5\% (Czubaty, 2015). En 1795, les administrateurs prussiens du district de Poznan écrivent à l'administration centrale à Berlin qu' "une petite minorité des maires maitrise la langue allemande et encore moins ne savent lire et écrire même le polonais [...] un problème que dans la génération actuelle et peut-être même dans celle à venir ne va guère être surmonté " ${ }^{2 .}$ Ces taux d'alphabétisation sont inférieurs à ceux obtenus par le comptage des signatures au moment du mariage par l'enquête Maggiolo (Furet, Ozouf, 1977). Relativement bien connu pour la France, coupée, grossièrement, en deux par la fameuse ligne Maggiolo, le degré d'alphabétisation est beaucoup moins bien établi pour l'Espagne de la fin du XVIII ${ }^{\mathrm{e}}$ siècle (Saugnieux, 1985). 
Plusieurs facteurs sont traditionnellement invoqués pour expliquer les progrès inégaux de l'alphabétisation en Europe. On reconnaît généralement un rôle au protestantisme dans la diffusion de la lecture et de l'écriture, expliquant en partie les disparités au sein de l'Europe. Les faibles taux d'alphabétisation en Europe centrale et orientale seraient dus à l'appauvrissement de la paysannerie réduit au "second" servage dans le cadre d'une économie domaniale fondée en grande partie à la fois sur l'emploi de paysans corvéables et de journaliers sans terre (François, 1989). En effet, l'effort de mettre en place une école élémentaire résultait davantage d'initiatives locales que de lois imposées par les États princiers. Par ailleurs, il a été établi depuis quelque temps que le «mouvement » des Lumières a largement dépassé le cadre des cercles intellectuels. En Allemagne, ce mouvement est connu sous le nom de "Lumières populaires" (Volksaufklärung) dans lequel de nombreux pasteurs, médecins, administrateurs et écrivains se sont engagés pour diffuser des connaissances dans les couches populaires (Böning, Schmitt, Siegert, 2007).

6 Les études menées récemment sur le rôle du maître d'école au sein des communautés villageoises dans les dernières décennies de l'Ancien Régime français (Simien, 2017) font apparaître des similitudes frappantes avec la situation en Prusse, étudiée il y a quelques décennies (Neugebauer, 1985). Indépendamment d'un cadre supérieur étatique ou ecclésiastique qui se veut contraignant, les communautés villageoises s'emparent du maître d'école, qui occupe de multiples fonctions et qui obéit aux configurations du pouvoir local au sein de sociétés villageoises qui, on le sait depuis longtemps, sont tout sauf homogènes, avec des variations géographiques à multiples échelles.

7 Davantage connus par leurs projets, qui rendent compte d'un état d'esprit et indirectement d'un rapport de force social, que par la mise en œuvre de ceux-ci, les illustrados espagnols réfléchissent également à la réforme des écoles élémentaires. Ils doivent cependant tenir compte de l'emprise exercée par le clergé catholique sur l'école. Les projets élaborés sous le règne de Charles III pour l'enseignement primaire semblent plutôt refléter l'acceptation d'un statu quo préservant le rôle des religieux et de la religion dans l'enseignement élémentaire. Mais l'éclatement de la Révolution française provoque aussi, comme dans d'autres pays, un durcissement de la censure et de la répression dans les monarchies voisines de la France, dont les gouvernements et les élites traditionnelles craignent la contagion révolutionnaire. Ce contexte est défavorable au développement de projets de réforme scolaire innovants (Aymes, 1985).

\section{B. Des défis inégaux à l'époque révolutionnaire}

8 De nombreux auteurs sont d'accord pour admettre que les acteurs de la Révolution avaient la conscience d'être à l'origine d'une histoire nouvelle qui impliquait la régénération de la société. Cette nouvelle société exigeait la création d'un homme nouveau en transformant le sujet, déformé par le joug du despotisme, en un citoyen libre et conscient de ses droits (Simien, 2017). L'école devait donc jouer un rôle central dans cette transformation de l'homme. Le résultat de la politique scolaire des gouvernements révolutionnaires a été réinterprété récemment. Traditionnellement, on interprétait le développement massif des écoles privées en France et l'échec des écoles publiques durant la décennie révolutionnaire et les suivantes comme le signe d'un conservatisme des communautés réfractaires au projet républicain. Mais de fait, les 
écoles privées qui se multiplient, ne sont autres que les écoles de communautés locales qui reprennent les pratiques d'autogestion de l'Ancien Régime et donc constituent des écoles publiques d'échelle locale (Simien, 2017). Le contenu et la mise en oeuvre de ces projets de régénération de la société par l'école firent cependant l'objet d'intenses débats durant les premières années de la Révolution (O'Connor, 2017, p. 10).

La foi affichée des révolutionnaires en la vertu de l'école pour la régénération de la société semble avoir trouvé un terreau particulièrement fertile aux Pays-Bas à l'époque de la République Batave puis du royaume de Hollande (1806-1810). Comme dans d'autres pays européens, l'éducation était un important sujet de débat aux Pays-Bas. Les élèves devaient désormais s'épanouir et non pas être matraqués de connaissances. Mais au contraire d'autres pays, l'accent y fut mis dès le départ sur l'enseignement primaire. Si cet enseignement primaire néerlandais était l'objet d'admiration d'observateurs extérieurs, aux yeux des réformateurs bataves, il s'agissait de surmonter ce qu'ils percevaient comme une grave crise économique et morale. Surtout l'unité et l'indivisibilité de la nation était considérée comme un enjeu, comme en France (Van den Burg, 2010). En effet, les transformations politiques depuis la révolution batave avaient été caractérisées par l'affrontement entre conceptions centralistes unitaires et fédéralistes partisanes d'une décentralisation.

Les projets d'envergure d'éducation de l'homme par l'école n'étaient pourtant pas propres aux États entrés en révolution. À l'époque révolutionnaire, on observe des tentatives d'une assimilation pragmatique mais systématique des populations polonaises intégrées dans l'État prussien après le dernier partage de la Pologne. La politique de développement économique menée par les administrateurs prussiens (Simsch, 1985) se double d'une politique de scolarisation, visant à germaniser la population polonaise. En effet, la multiplication des impôts par quatre dans la nouvelle province de Prusse méridionale (fig. 1), anciennement polonaise, s'accompagne aussi d'un programme ambitieux de construction d'écoles suivant un plan visant à permettre aux villages des domaines royaux comme nobiliaires d'accéder à une école. Ainsi dans le département administratif de Poznan (Posen), la construction des bâtiments d'école fut réalisée suivant plusieurs plan (fig. 2 a et b). Les administrateurs prussiens reconnaissaient tout à fait la valeur des projets scolaires conçus par le gouvernement polonais pendant la décennie précédant la disparition de l'État polonais, comme le règlement scolaire de 1783, complétée en 1790, la mise en place d'une commission d'éducation nationale et la reconnaissance d'un ordre particulier, celui des « académiciens ». Mais, peut-être aussi pour mieux mettre en valeur les efforts de l'État prussien, ils soulignaient la faiblesse du réseau scolaire dans la nouvelle province: dans les 9166 villages de la province, il n'y aurait eu que 489 écoles, soit un peu plus de $5 \%$ des villages (Klewitz, 1805). A titre de comparaison, dans les provinces prussiennes centrales, très majoritairement germanophones et protestantes, entre 80 et $100 \%$ des villages disposaient d'une école (Neugebauer, 1985). Le plan de construction conçue en 1800 pour les provinces anciennement polonaises, a abouti à la construction de 255 écoles, alors que 279 autres étaient en construction, ce qui aurait donc conduit à un doublement de la densité du réseau scolaire en 5 ans. 
Document 1 :

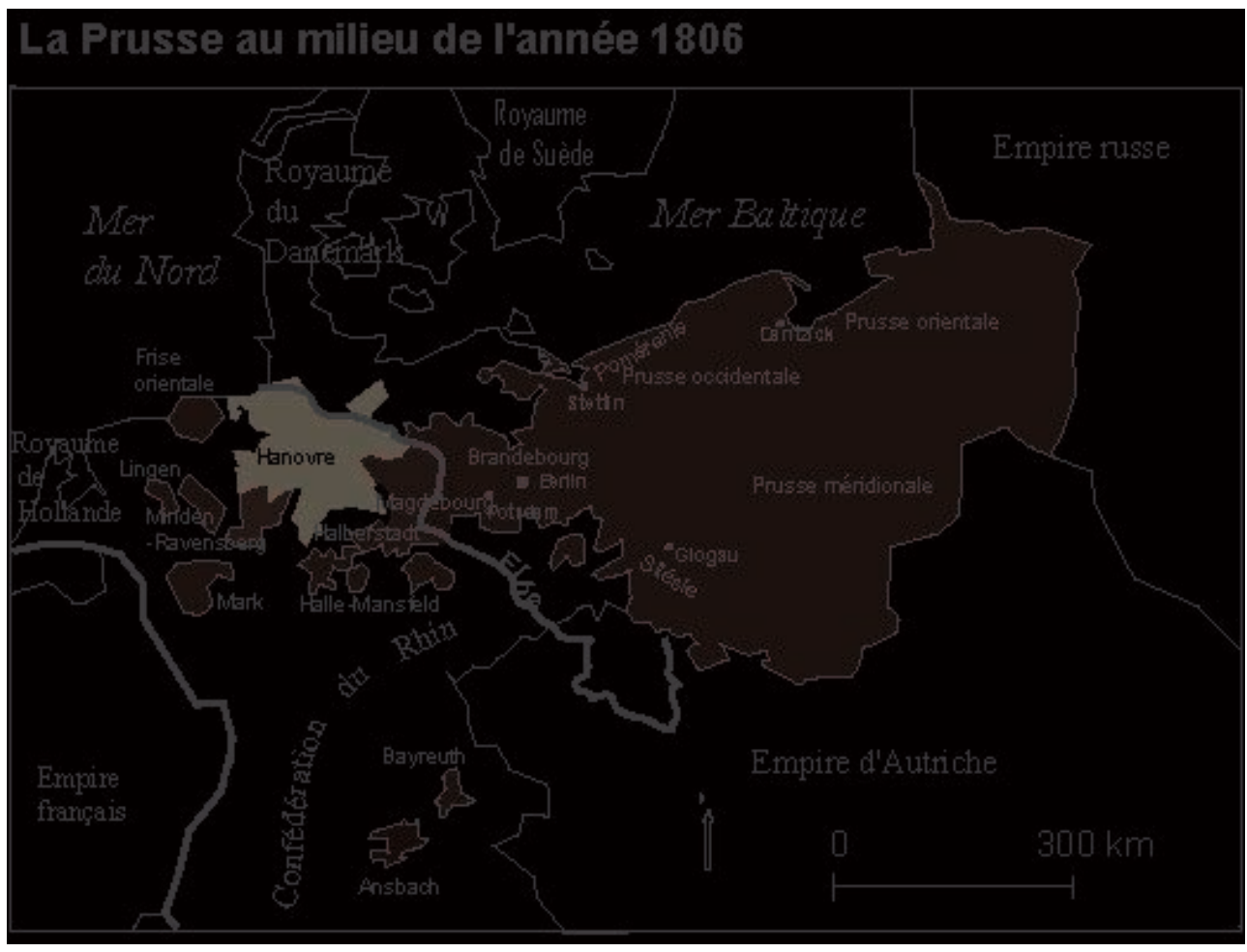

Document 2 a Dessin d'une école rurale en Prusse méridionale Archiwum Główne Akt Dawnych (AGAD), GD Südpreussen, 1601)

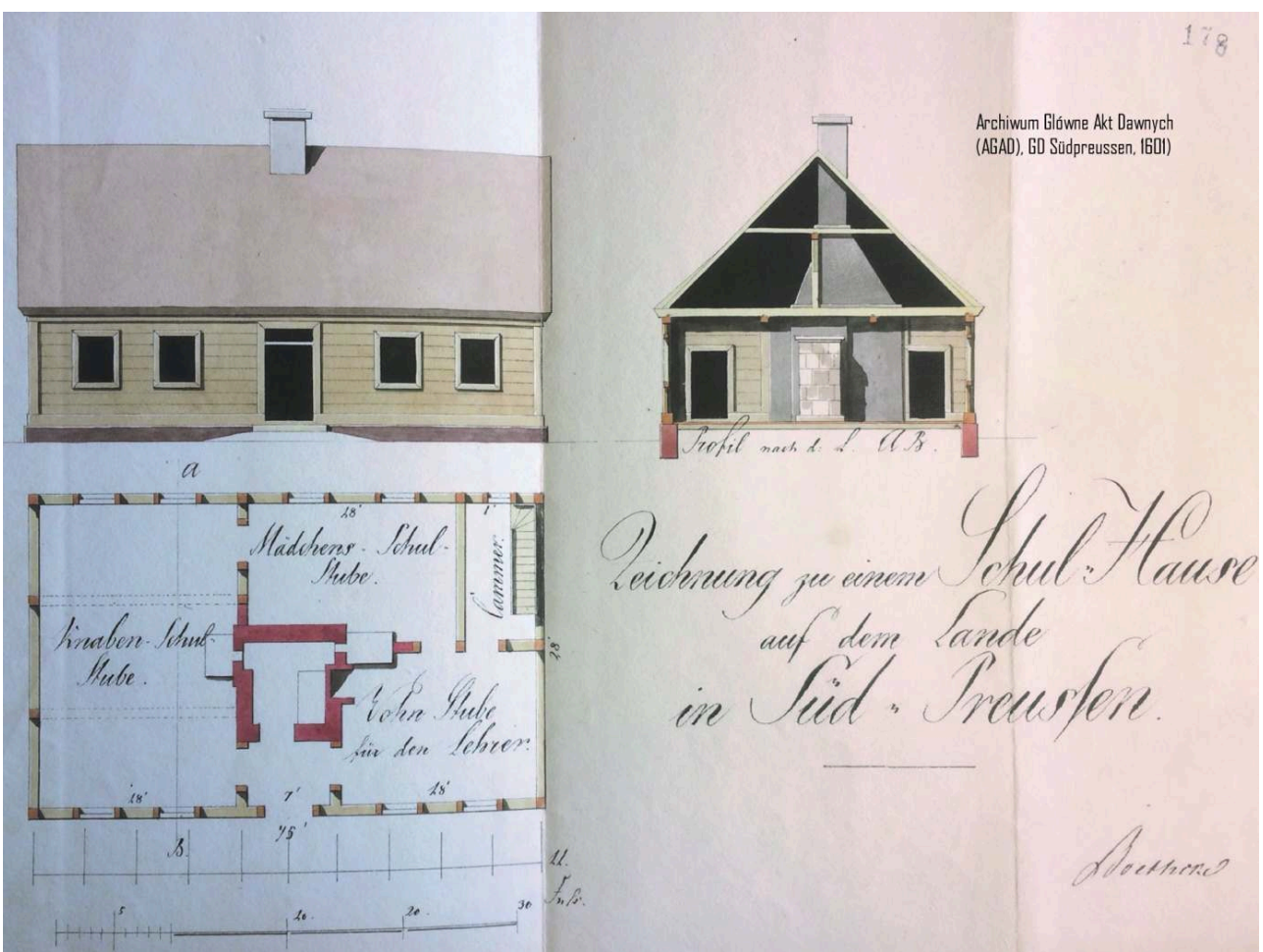

Plusieurs plans furent conçus pour les bâtiments d'école en Prusse méridionale. Celuici prévoyait des salles de classes séparées pour filles et garçons, ce qui était le reflet de contenus d'enseignements différents. 


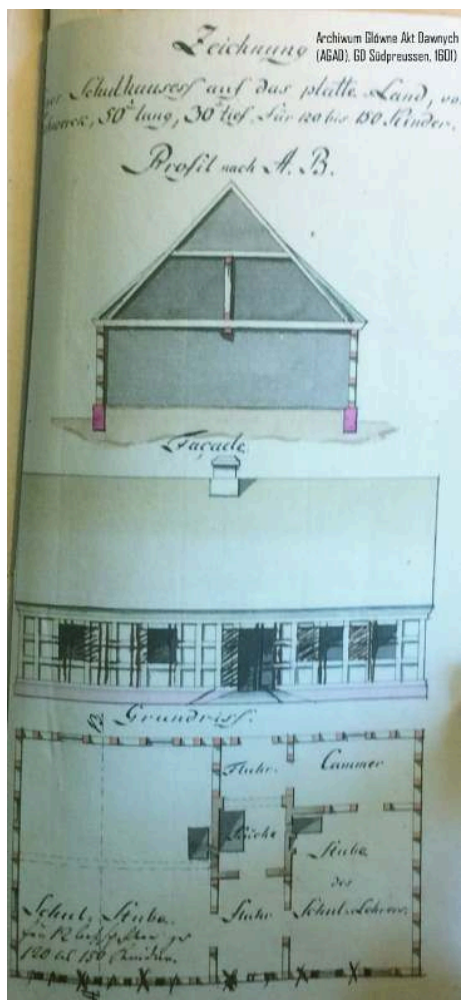

(AGAD, GD Südpreussen, 1601)

Mais cette politique visait à faire des habitants de la nouvelle province de fidèles sujets $\mathrm{du}$ roi de Prusse. L'enseignement dans les écoles rurales devait se limiter à l'apprentissage de la lecture, de l'écriture de la langue allemande et polonaise, au calcul et à la religion. Il devait être à la fois enseignement et éducation morale. Mais dès que l'enseignement de la religion passerait à l'étude des "particularités d'une confession particulière » (sous-entendue le catholicisme), il reviendrait à l'ecclésiastique du lieu d'en assumer la responsabilité, et non au maître d'école, qui ne devait enseigner la religion qu'en général ${ }^{3}$. En effet, le livre de lecture contenait un court chapitre consacré à Dieu, présenté comme l'être suprême, précédé d'un chapitre expliquant l'utilité de l'autorité à laquelle tout le monde devait obéir. Ce qui, à première vue, pourrait apparaître, comme un compromis dans une monarchie devenue davantage multiconfessionnelle par l'intégration de populations catholiques importantes, visait en réalité à assimiler ces nouvelles populations. La « géographie » de ce livre de lecture se limitait à un paragraphe de 5 lignes :

"Avec nous sont encore les habitants de nombreux villes et villages sous un maître suprême, le Roi. Toutes les provinces que commande notre Roi sont appelées l'État prussien. Il y a encore beaucoup d'autres pays et États. »

Il ressort des discussions menées par les administrateurs prussiens au sujet du manuel élaboré particulièrement pour cette province que le maître d'école devait étudier avec les garçons le dimanche la partie du manuel consacrée à la connaissance de la " constitution " prussienne. Par ailleurs, au cas où le maître d'école ne maîtriserait pas la langue allemande, mais bien le curé ou le vicaire, ceux-ci devraient utiliser les heures revenant au curé (donc le temps normalement prévu pour l'enseignement religieux) à 
l'apprentissage de l'allemand. L'enseignement de la religion catholique ne devrait commencer qu'après l'acquisition de "ces bases générales ». Le guide pédagogique à l'usage des maîtres d'école en langue allemande comme polonaise, ne devait être communiqué au maître d'école qu'au cas où celui-ci ne maîtriserait pas l'allemand. S'il était Polonais, mais avait des rudiments de la langue allemande, la version polonaise ne devrait pas lui être transmise. En d'autres termes, insensiblement, l'allemand devait remplacer le polonais. Les administrateurs prussiens se plaignaient de l'absentéisme des élèves et l'un d'entre eux allait jusqu'à proposer le châtiment corporel pour les parents de ces enfants ! Si cette proposition ne fut pas retenue, elle témoigne néanmoins de l'esprit qui animait certains de ces administrateurs.

\section{L'entrée de la géographie dans l'enseignement primaire}

\section{A. Combattre le « localisme » des ruraux au profit de l'État princier}

Si l'école élémentaire se consacrait donc essentiellement à l'apprentissage de la lecture, de l'écriture, du calcul et de la morale, la géographie entrait néanmoins lentement dans le cercle des disciplines enseignées. En France, des indications sur l'enseignement de la géographie et de l'histoire étaient contenues dans le décret de 1794 (Clerc, 2014), mais seulement les instructions de 1834 prévoyaient un enseignement de géographie à partir de l'âge de 10 ans (Furet, Ozouf, 1977). Les révolutionnaires estimaient que la participation active à la vie politique exigeait davantage que la connaissance par les citoyens des rouages institutionnels, elle nécessitait aussi des connaissances de base en géographie (O'Connor, 2017). Il semblerait pourtant que ce soient les États allemands, appartenant d'abord au Saint Empire Germanique (jusqu'en 1806), puis à la Confédération du Rhin (1806-1813), et enfin la Confédération Germanique (1815), qui aient été les premiers à intégrer la géographie dans les savoirs élémentaires, si l'on excepte les Pays-Bas. Les préoccupations du mouvement dits des «Lumières populaires » (Volksaufklärung) comportaient bel et bien une dimension géographique, notamment pour ce qui était du choix de l'échelle spatiale de l'identité territoriale. Les prédicateurs protestants des villages auraient éprouvé, au XVIII ${ }^{\mathrm{e}}$ siècle, un sentiment d'abandon de la part des autorités princières et des élites urbaines. Ces prédicateurs reprochaient souvent aux ruraux, auxquels la culture urbaine émergente les assimilait, le manque d'intérêt pour la communauté suprarégionale, fustigeant leur localisme égoïste (Haussmann, 1999, p. 207). Que cette critique corresponde à une analyse adéquate de la situation est certainement discutable. Les communautés paysannes défendaient leurs intérêts face aux seigneurs dans un État qui faisait peser sur elles l'essentiel des charges publiques comme le service militaire ou les contributions. Une solidarité territoriale dans le cadre l'État princier tel que la Prusse, la Saxe ou la Bavière devait leur apparaître comme une exigence abstraite. Mais leur horizon géographique ne se limitait pas à celui perceptible depuis le clocher de leur village. Toujours est-il qu'en 1794, Karl Traugott Thieme publia un livre de lecture à destination des écoles rurales et urbaines d'une principauté particulière, la Saxe électorale, intitulé Gutmann ou l'ami de l'enfant saxon (Thieme, 1794).

16 Comme les livres de lectures qui l'avaient précédé, L'ami de l'enfant saxon contenait de nombreuses séquences organisées sous forme d'histoires et de dialogues entre le maître 
et les écoliers. Mais alors que L'ami de l'enfant. Un livre de lecture pour les écoles de campagne de Rochow (Rochow, 1776), publié en 1776 contenait deux séquences dédiées à ce que l'on pourrait appeler la physique du globe terrestre et l'astronomie et à peine une demie page évoquant les continents, L'ami de l'enfant saxon de Thieme est divisé en 118 petits chapitres ou séquences, dont une est consacrée à la surface de la Terre (le planisphère), une à la signification du mot pays et les deux séquences suivantes aux provinces de la Saxe électorale. En fait, la notion de " pays » n'est expliquée que par le seul exemple saxon (la Saxe électorale). D'autres Etats ne sont pas mentionnés et n'apparaissent que sous la mention "étranger». Dans le cas d'un district presque enclavé dans du territoire prussien, le nom de "Prusse » ou de «Brandebourg » semble avoir été soigneusement évité. Au moment de la Révolution française et une douzaine d'années avant la disparition du Saint Empire Germanique, Thieme ne voyait donc manifestement aucune raison pour évoquer le cadre du Saint-Empire ou une entité « allemande » quelconque. Si l'on admet que les petites gens étaient attachées d'abord au cadre local, ensuite dans un horizon plus lointain, à la principauté, et en dernier lieu, au cadre du Saint-Empire, un " patriotisme impérial » populaire aurait cependant subsisté dans le dernier tiers du XVIII ${ }^{e}$ siècle, selon certains auteurs. Les sources, à savoir les requêtes faites à la cour de justice de l'Empire, souvent contournant la justice locale ou celle de leur principauté, permettant de démontrer l'existence de ce cadre de référence dans l'esprit des couches sociales inférieures, révèlent cependant toute l'ambiguïté du concept d'identité territoriale, car ces requêtes n'étaient évidemment pas désintéressées (Noël, 2013). L'attachement identitaire à un territoire était variable en fonction des enjeux individuels et collectifs. Quelques années plus tard, à l'époque de la domination française, des communautés paysannes, parfois appuyées sur de vastes réseaux dans les États satellites créés par Napoléon, s'adressent directement à l'Empereur des Français, pour contourner la justice de leur État, qu'ils jugent de connivence avec les anciens seigneurs (Severin-Barboutie, 2008, Todorov, 2011).

\section{B. L'impulsion donnée par la période révolutionnaire et napoléonienne aux contenus de l'enseignement de la géographie - le rôle de l'Allemagne}

Dans le sillage de la Révolution, la conquête napoléonienne conduit à une réorganisation du territoire du Saint-Empire par le Recès de 1803, faisant disparaître 110 petits territoires. La fin du Saint-Empire en 1806 et la création de la Confédération du Rhin ainsi que son élargissement en 1807 continuent à remodeler la carte de l'Allemagne. Les États ayant soutenu la France voient leurs territoires s'agrandir alors que Napoléon crée des États tout-à-fait nouveaux en rassemblant des provinces conquises, tels que le royaume de Westphalie ou le grand-duché de Berg. L'effort de légitimation de ces nouveaux Etats semble avoir été soutenu par ceux qui souhaitent depuis des décennies inculquer aux populations rurales une identité territoriale dépassant le cadre du village.

Ainsi, dans l'article "De l'éducation du peuple des campagnes au patriotisme par l'enseignement scolaire " publié en 1810, le prédicateur de Felsberg près de Cassel, capitale westphalienne (dans l'ancien électorat de Hesse) Karl Christian von Gehren (Gehren, 1810) discute l'utilité du patriotisme qu'il définit non pas comme un attachement à une nation, un territoire ou une langue mais comme un attachement de 
l'individu à ses concitoyens, à la constitution de son pays et à son prince (Landesherrschaft), un attachement dont découlerait la conviction que ces choses-là étaient véritablement dignes d'amour et de respect. Après un passage consacré au patriotisme allemand, qui laisserait penser à des intentions subversives (c'est-à-dire nationalistes allemandes et donc antinapoléoniennes), l'auteur déplore l'absence de manuels faisant connaître à la population rurale leur pays, une connaissance qui serait un prérequis pour l'amour de la patrie. Il conclut alors :

«Il faudrait s'occuper avant tout d'un livre de lecture adapté aux besoins des enfants du plat pays pour éveiller et promouvoir leur sens patriotique, un livre qui tiendrait compte des particularités de tel ou tel pays, auquel il serait destiné, par exemple le Royaume de Westphalie ».

L'absence de ce genre de livre s'expliquerait par l'incertitude et l'instabilité politique. Mais désormais, en 1810, ces changements de régime seraient près de toucher à leur fin :

« en tous cas, il y a un besoin urgent pour une nation comme la royalwestphalienne et pour toute autre nation allemande qui se réjouit d'une constitution stable d'éveiller et de faire vivre le sens patriotique en son sein, et par conséquent de réaliser un manuel pour la propagation de celui-ci à l'usage des écoles villageoises ".

Officiellement, l'auteur souhaitait donc inculquer aux enfants un sentiment identitaire territorial correspondant aux différents Etats allemands.

Ce livre devrait contenir une présentation topo- et géographique du territoire pour lequel il serait écrit ainsi qu'une explication brève des lois, de la constitution, des notions d'autorité, de citoyenneté, de patrie. Bref, le prédicateur westphalien dans l'ancienne Hesse, invite ses lecteurs à écrire un manuel de géographie et d'éducation civique ou plutôt un livre de lecture contenant des chapitres consacrés à ces matières, destiné à donner aux jeunes sujets du roi Jérôme une identité territoriale collective et un patriotisme spécifiquement westphalien. En principe, il invite à adapter les livres de lecture aux besoins spécifiques de tous les Etats allemands, réduits en nombre depuis 1803 et 1806, en suivant, sans s'y référer explicitement, le modèle de L'ami de l'enfant saxon de 1794. L'auteur, qui avait été acquitté de l'accusation d'avoir participé activement aux petites révoltes ayant tenté de renverser le régime en 1809, ne cherchait peut-être qu'à montrer sa loyauté au gouvernement. Mais son appel fut entendu. En 1810, d'autres pasteurs westphaliens s'adressent au ministre de l'Intérieur pour obtenir l'autorisation de publier un almanach populaire, utilisant des arguments similaires à ceux déployés par Gehren (Paye, 2013). Celui-ci, lavé de l'accusation d'avoir participé à l'insurrection de 1809, devait se méfier de la surveillance policière, d'autant plus qu'il venait d'être libéré, pour la troisième fois et dispensé de l'obligation de se présenter toutes les semaines à la police de Cassel. Même dans son récit de ses incarcérations, publié en 1815 , où il exalte un patriotisme allemand et hessois gallophobe, il n'avouera ni une participation active à l'insurrection de 1809 , ni une activité conspirative ultérieure quelconque (Gehren, 1816).

Quelles que soient les véritables intentions de l'auteur, le manuel désiré par Gehren voit effectivement le jour l'année suivante (1811), rédigé par le fils du célèbre Volksaufklärer Heinrich Gottlieb Zerenner. Publié par Carl August Kümmel à Halle, l'éditeur du Journal für Prediger, ce livre, L'ami de l'enfant westphalien répond aux souhaits formulés par von Gehren. Carl Christoph Gottlieb Zerrenner, prédicateur à Magdebourg, présente les deux chapitres consacrés à la géographie et à l'éducation 
civique comme la nouveauté qui distinguerait son livre d'autres livres de lecture, dont les réalités seraient alors devenues étrangères à la population. Dans son cours consacré aux lois et aux institutions de l'État westphalien, le prédicateur explique la nécessité de l'autorité étatique par le besoin de protection des faibles, sans la moindre référence à la volonté divine. Mais il explique aussi aux enfants l'honneur qu'aurait fait le grand homme au peuple westphalien en faisant son frère roi. Le plus grand crime qu'on puisse commettre consisterait à attenter à la vie du roi. La moitié du cours de géographie est consacrée au royaume de Westphalie (fig. 3). La description de ses villes met en valeur le rôle du nouveau centre politique Cassel (fig. 4). L'usage patriotique de la géographie faisait aussi partie de la propagande napoléonienne (Lignereux, 2014). Sans critiquer la démarche ouvertement, la critique littéraire allemande rejette le livre dont les deux chapitres seraient anti-pédagogiques.

Document 3 : La place de la Westphalie dans le cours de géographie de Zerrenner

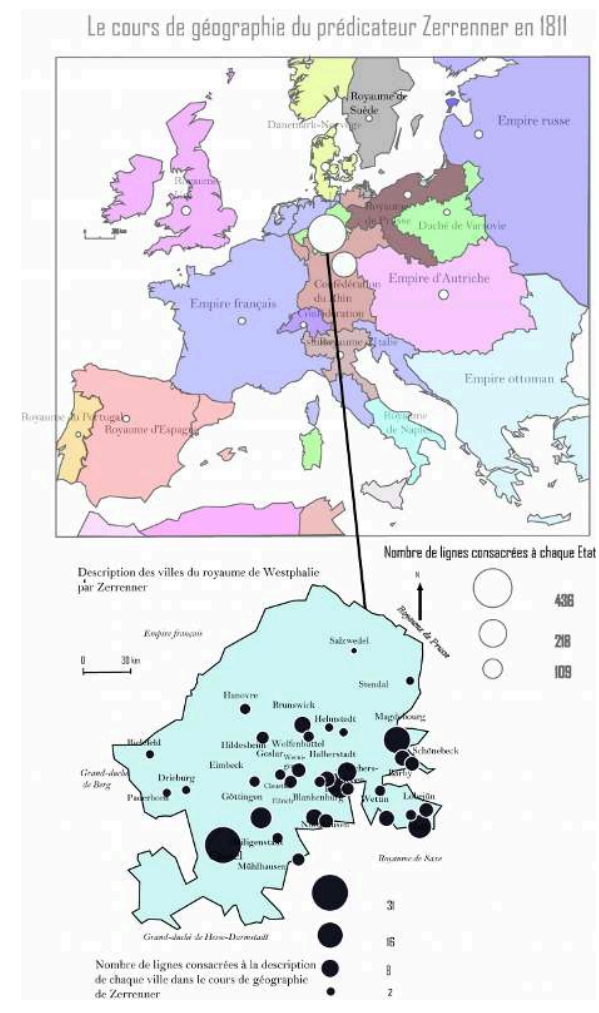


Document 4 : La géographie de la Westphalie d'après le prédicateur Zerrenner

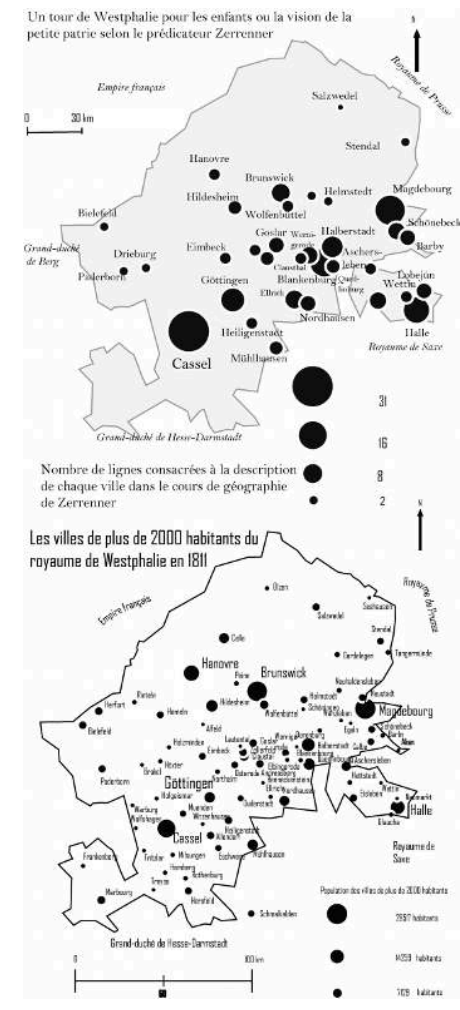

23 Né près de Magdebourg, Carl Christoph Gottlieb Zerrenner décrit d'une manière détaillée les lieux qu'il fréquente et qu'il a visités : la ville de ses études, Halle, et le nouveau centre politique, Cassel. Il reste vague au sujet du Nord du royaume, sans doute en raison des modifications territoriales de l'année 1810. L'importance accordée à chaque ville ne correspond donc pas à sa place réelle dans la hiérarchie démographique, administrative et économique, mais reflète davantage son rôle culturel: Göttingen éclipse Brunswick.

Le manuel de Zerrenner dans sa version initiale n'a sans doute guère connu de large diffusion. Les défaites militaires françaises mettent fin à l'expérience d'entités étatiques napoléoniennes et les maîtres d'école du royaume de Westphalie, s'ils avaient été favorables à l'utilisation de ce livre de lecture, n'auraient guère eu le temps de l'expérimenter avec leurs écoliers. Il n'en était sans doute pas de même de la deuxième version du livre, rédigée après le retour dans le giron de la monarchie prussienne. Les mêmes pasteurs qui avaient célébré le roi Jérôme Bonaparte et nommé Napoléon «le grand homme ", célébrèrent la victoire et le retour du roi de Prusse dès novembre 1813. Zerrenner, qui avait cherché à inculquer aux enfants westphaliens l'amour du roi Jérôme, se met immédiatement à réécrire son livre de lecture, renommé L'ami de l'enfant allemand.

La critique littéraire est acerbe :

« Le Westphalien est devenu un Allemand et tout ce qui a été imposé au premier par l'époque pendant laquelle et pour laquelle il était écrit est devenu étranger et hostile au second. Il laisse derrière lui, Dieu merci, tout ce qui rappelle ces années passées de la servitude, y compris ce petit mot fâcheux, précocement disparu du titre $»^{4}$ 
Dans les deux chapitres consacrés à la géographie et à l'éducation civique, toute référence à la Westphalie est supprimée. La description des villes est étendue à l'ensemble de l'Allemagne et ne suit plus l'ordre qui laisserait apparaître un regroupement géographique rappelant le royaume de Westphalie. Mais surtout, cette nouvelle version, de même que celle de L'ami de l'enfant bavarois publié en 1816, qui apparaît comme une réponse au livre de Zerenner, font apparaître à la fois l'héritage et la nouveauté issus des bouleversements de la période de 1789 à 1815 : alors que l'État princier - comme la Bavière ou la Prusse - occupe beaucoup plus d'espace dans les chapitres destinés à la géographie que les autres États allemands individuellement, indépendamment de leur taille ou de leur poids démographique, le cadre allemand, formé par l'ensemble des autres États allemands, est plus amplement décrit. Les rééditions de ces livres pendant les décennies suivantes (et l'épuisement rapide des stocks des premières éditions) laissent supposer qu'à côté d'une identité territoriale forte liée aux Etats composant la Confédération Germanique fondée par le Congrès de Vienne, une identité territoriale allemande a pu émerger ou se consolider à nouveau. Les autres continents se voient aussi attribuer un peu plus d'espace que dans L'Ami de l'enfant Westphalien, mais y voir une conséquence d'une réouverture au monde après la fin de la guerre maritime et le blocus continental serait sans doute pousser l'analyse un peu trop loin.

\section{Document 5 :}

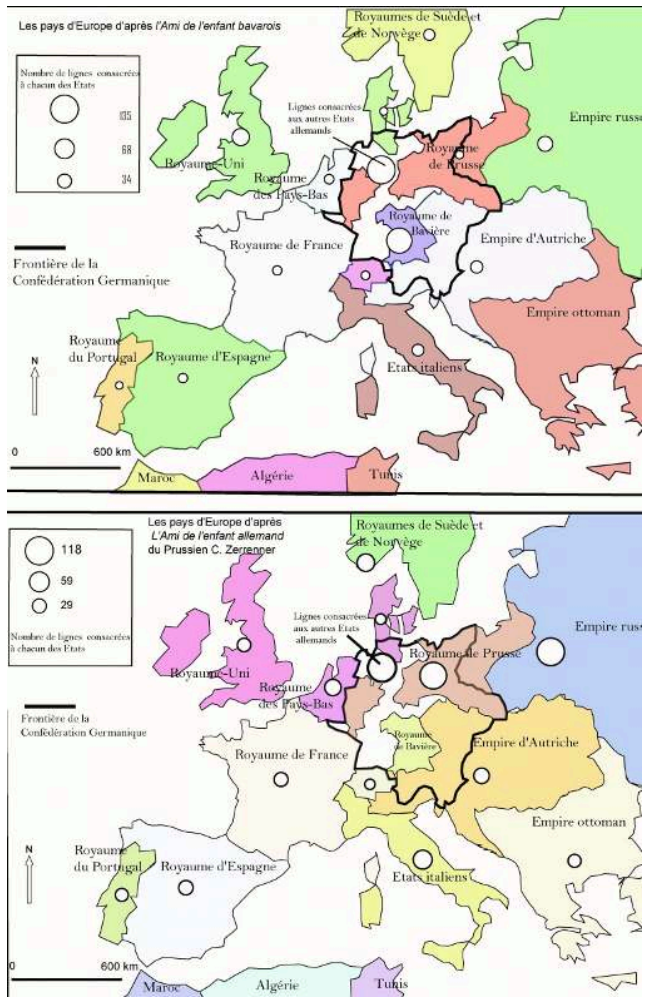

L'espace consacré aux différents pays d'Europe dans les chapitres de géographie des livres de lecture de L'Ami de l'enfant bavarois (Mayer, 1816) et de L'Ami de l'enfant allemand du Prussien Zerrenner (1815). Dans les deux cas, le royaume auquel appartient l'auteur, que ce soit la Bavière ou la Prusse, occupe une place de choix dans la description géographique, mais dans les deux cas, les autres Etats composant la 
Confédération Germanique, dans leur ensemble se voient traités plus amplement que l'État princier, à la différence du chapitre de géographie de L'Ami de l'enfant westphalien (Zerrenner, 1811) nettement centré sur la Westphalie ou de L'Ami de l'enfant saxon, qui n'évoquait que la Saxe (Thieme, 1794).

Les bouleversements révolutionnaires se répercutèrent aussi sur politique scolaire d'autres pays et la réflexion pédagogique. Mais la géographie ne semble guère faire l'objet de développements particuliers. En Espagne, Francisco de Cabarrús republie, en 1808, ses Cartas sobre los obstáculos que la naturaleza, la opinion y las leyes oponen a la felicidad publica qu'il avait adressées déjà en 1795 au Prince de la Paix, Godoy, après la conclusion de la paix entre l'Espagne et la France révolutionnaire. La géographie comme discipline enseignée n'y est pourtant que brièvement mentionnée. Il évoque des écoles de géographie, mais dans son esprit, il s'agissait d'écoles secondaires. Dans les écoles élémentaires, il s'agirait, selon lui, d'apprendre aux enfants à lire, à écrire, à compter et les premiers éléments de la géométrie pratique. Mais à l'égal de Zerrenner, il préconise d'enseigner un catéchisme politique, qui comprendrait la présentation de la société dans laquelle l'enfant vivait et les bénéfices qu'il tirerait d'elle. Il s'agirait d'une sorte de cours d'éducation civique, comme celui de L'Ami de l'enfant westphalien. Pour Cabarrús, ce catéchisme politique devrait contribuer au bonheur national et serait plus utile que l'enseignement des «principes abstraits de la théologie».

Le moindre intérêt pour l'enseignement de la géographie à l'école élémentaire résultait peut-être d'un sentiment national espagnol bien plus affirmé que celui des Allemands, en dépit des particularismes de la monarchie espagnole que les efforts des Bourbons au cours du XVIII ${ }^{e}$ siècle n'avaient pas réussi à effacer. Peut-être aussi la délimitation physique plus nette de l'Espagne péninsulaire, séparée de son voisin septentrional par la chaîne des Pyrénées, n'incitait guère à justifier les frontières par un enseignement de la géographie. Toujours est-il que l'auteur préconise au sujet des écoles techniques le recours à des gazettes mensuelles et l'établissement de jardins botaniques afin de familiariser les élèves avec les différentes provinces de l'Espagne, dont certaines feraient comprendre toutes les « nuances du climat de notre péninsule ».

30 A l'est, après la création du duché de Varsovie (1807), que les Polonais perçoivent comme une première étape vers la résurrection de la Pologne, le conseil de gouvernement de ce duché s'emploie à poursuivre le programme de construction d'écoles, mais donne aussi l'impulsion à une réflexion pédagogique. Un enseignement de géographie dans les écoles élémentaires polonaises n'apparait pourtant guère comme une préoccupation dans les débats menés au sujet des contenus d'enseignement et la formation des maîtres d'école. Et le projet de Wojciech Izydor Choynacki d'étendre l'enseignement élémentaire entre autres à la géographie, postérieur à l'existence du duché (1815), reste subordonné à la formation morale et religieuse de l'enfant (Winiarz, 2016). Car, comme aujourd'hui, l'enseignement de la géographie ne commençait qu'après l'apprentissage de la lecture.

\section{Comment enseigner la géographie aux écoliers?}

Dans l'état de nos connaissances, les pratiques pédagogiques réelles de l'enseignement de la géographie ne se laissent deviner que par le contenu et la structure des manuels ou encore des écrits de pédagogues. Les premiers manuels de géographie à destination des élèves des écoles primaires proprement dits semblent avoir été publiés aux Pays- 
Bas. Dans sa brochure intitulée Les tout débuts de la géographie publiée en 1809, H. C. Scheerder consacre seulement trois des quinze pages au royaume de Hollande et à ses subdivisions administratives. Le livret se résume à des questions immédiatement suivies des réponses. La Géographie pour enfants à l'usage des écoles, publiée par Pieter Nicolaas Muyt en 1814 (Aardrijksbeschrijving voor Kinderen, ten Gebruike der Schoolen) suit la démarche inverse. Ce livre d'une cinquantaine de pages est divisé en 10 chapitres, commençant par la description du globe terrestre, les planètes, puis sont abordés les cercles de la Terre, les différentes masses d'air. Seulement le chapitre 8 est consacré aux continents, mis à part l'Amérique, qui bénéficie des deux derniers chapitres, l'un consacré à sa découverte, l'autre à sa description. Les Pays-Bas ne sont pas traités directement, ce qui peut être le reflet de l'incertitude sur son avenir politique au moment de la rédaction de l'ouvrage. Le royaume de Hollande avait en effet été annexé à la France impériale en 1810 et au moment de la parution, les troupes françaises venaient d'évacuer le pays alors que le royaume des Pays-Bas ne fut proclamé qu'en mars 1815. Il est probable qu'une deuxième partie, consacrée à la géographie politique, était prévue. Tous les chapitres du petit manuel sont suivis d'un questionnaire destiné à vérifier ce que les élèves ont retenu et compris.

Dans son Ami de l'enfant westphalien, Carl (ou Karl) C. G. Zerrenner cherche à inculquer aux enfants la connaissance d'un territoire de création récente, d'une entité politique nouvelle, fondée après le traité de Tilsit (1807). Comme on le connaît d'autres manuels scolaires plus récents, la connaissance du territoire de l'État proche occupe une part essentielle du cours de géographie de Zerrenner. La manière dont le théologien Zerrenner présente la géographie aux écoliers reflète le stade de l'évolution de la science géographie de son époque : c'est une géographie humaine plus préoccupée par l'inventaire des lieux que de l'espace, comme on a pu écrire (Laboulais-Lesage, 2001), même si Conrad Malte-Brun se proposait déjà de considérer davantage les éléments faisant l'unité d'un espace en géographie humaine à l'instar de ce que Humboldt pratiquait dans la géographie physique (Malte-Brun, 1810). De fait, les descriptions comprennent tout de même des remarques sur les caractéristiques représentatives d'un pays. La Suède est ainsi présentée comme un pays boisé, les principales productions des pays sont mentionnées... Zerrenner est d'ailleurs un lecteur attentif des publications de Carl Ritter. Zerrenner énumère les villes les unes après les autres et les décrit avec plus ou moins de détails, apparemment, selon ses connaissances personnelles. Les villes où il a vécu ou fait ses études comme Halle bénéficient d'une présentation plus détaillée que les villes du Nord du royaume. Il n'est pas impossible que l'instabilité des frontières du royaume se soit répercutée sur l'importance accordée aux différentes parties du royaume. Après le rattachement des parties septentrionales de l'électorat de Hanovre au royaume de Westphalie pendant quelques mois en 1810, Napoléon décide d'en retirer la partie littorale au royaume de son frère, d'où peut-être le peu d'espace consacré au nord du royaume, il est vrai, moins urbanisé (fig. 4).

Il n'a guère le souci de justifier l'unité de l'espace, sa particularité par rapport à d'autres États. Justifier l'unité territoriale du royaume de Westphalie, composé de 29 provinces et territoires conquis, assemblés par la volonté de son créateur, aurait sans doute été un exercice difficile, par ailleurs incompréhensible aux élèves. En effet, ni sur le plan de la géographie physique, ni sur celui de la géographie humaine, le royaume présentait d'homogénéité qui aurait permis de légitimer, aux yeux des enfants, l'assemblage de ces provinces. Mais quelques années plus tard, le même Zerrenner, 
dans son guide à destination des maîtres d'écoles, insiste sur la nécessité d'enseigner les éléments qui font l'unité d'un espace. Mais comme à cette époque, seule la géographie physique, commençait à s'intéresser aux caractéristiques communes d'un espace, il recommande de mettre l'accent sur le relief.

"Comme les frontières politiques des pays dans ces temps de l'instabilité sont si peu fixes, on mettra l'accent sur la situation des mers, des fleuves, des montagnes etc. et après sur la situation de certaines localités importantes. Lors des cours de géographie consacrés à l'Allemagne et des différents Etats allemands, dans lesquels nous vivons, on doit faire attention aux subdivisions principales, aussi le nombre de villes que l'on traite en cours. Mais on doit sélectionner celles qui ont une importance politique, historique, commerciale ou littéraire. Seulement pour la capitale et quelques villes très importantes, on indiquera le nombre d'habitants et des maisons et comparera ensuite les autres avec ces premières ». (Zerrenner, 1816)

Dans la géographie savante allemande, cette propension temporaire à valoriser la géographie physique aurait été la réaction à l'instabilité politique de la période révolutionnaire et napoléonienne, rendant la réalisation d'oeuvres de longue haleine illusoire (Garner, 2008). Le remodelage permanent des entités politiques a pourtant donné lieu à une importante production de descriptions topographiques et statistiques au service des États nouveaux et de leurs habitants. L'instabilité des frontières semble avoir davantage préoccupé les géographes scolaires, soucieux d'enseigner des vérités durables. Par ailleurs, l'intérêt pour des unités homogènes de l'espace aussi bien dans le domaine de la géographie humaine que physique reflétait le souci d'éviter la tentation d'exhaustivité. Les élèves devraient apprendre l'essentiel, mais l'acquisition du savoir devait se faire par la répétition fréquente.

" Le professeur cherchera lors de l'étude de la patrie à attirer l'attention particulièrement sur les établissements et institutions qui inculqueront à l'enfant l'amour de sa patrie et de son prince ». (Zerrenner, 1816)

Un paradoxe caractérisait pourtant cette géographie des écoles primaires naissante. Alors que l'enseignement primaire, ne serait-ce que par les récits que faisaient lire les maîtres aux enfants, était initialement centré des connaissances et savoir-faire pratiques utiles aux classes populaires et plusieurs auteurs commençaient leur chapitre de géographie en expliquant aux enfants l'utilité pratique de la géographie, les manuels et livres de lecture suivent généralement la démarche du global au local. Ce dernier devait pourtant paraître plus concret aux enfants.

Dans ses recommandations adressées aux maîtres d'école, Zerrenner insiste beaucoup sur la nécessité d'apprendre aux enfants à s'orienter. Les enfants devaient apprendre les points cardinaux tôt et les associer à des villes. «Dans quelle direction marcheraistu si tu devais aller à Berlin, à Paris ou à Saint-Pétersbourg ? Si tu devais gagner Berlin depuis Leipzig?» Les distances entre des villes importantes devaient être indiquées. Zerrenner insiste sur l'usage des cartes, à fixer sur un support rigide. Après avoir fait localiser aux enfants les villes sur les cartes, il faudrait enlever les cartes et demander de localiser les villes, montagnes, fleuves ... au tableau ou sur l'ardoise. Si les enfants ont quelques facilités à dessiner, on leur demanderait à recopier des cartes ou à en dessiner de mémoire. Mais les exemples de villes citées par Zerrenner montrent peutêtre aussi que l'horizon géographique des classes populaires au début du XIX ${ }^{\mathrm{e}}$ siècle, par les migrations de travail plus vastes qu'admis encore il y a quelques années, dépassait largement celui visible depuis le clocher du village. Peut-être aussi les 
circulations propres à la période révolutionnaire et napoléonienne par le départ de nombreux jeunes comme soldats, de même que les passages de troupes, ont contribué à élargir l'horizon des masses populaires, faisant apparaître les exemples de Zerrenner comme très concrets aux élèves. Ces enfants n'ont-ils pas eu des frères ou du moins entendus parler de soldats péris en ou revenus de Russie, vu des soldats de France et d'ailleurs?

\section{Conclusion}

C'est donc apparemment pendant la période révolutionnaire et napoléonienne (1789-1815) que la géographie a fait son entrée dans le cercle des disciplines enseignées à l'école primaire, mais d'une manière et avec une intensité spatialement très inégales. Il semblerait que la géographie soit entrée dans l'enseignement primaire d'abord dans l'espace allemand, si l'on excepte l'espace des Pays-Bas. En effet, les livres de lecture utilisés dans les écoles élémentaires allemandes sont les premiers à intégrer des séquences spécifiques dédiées à la géographie. Avant de proposer des explications sur cette précocité supposée, il faudrait cependant signaler que cette impression peut résulter d'une connaissance insuffisante de la littérature pédagogique européenne tant cette littérature était abondante et multilingue. Si nous n'avons pas repéré de livres de lecture contenant des chapitres consacrés à la géographie à destination des écoliers espagnols, italiens et polonais, il n'est pas impossible qu'il y en ait eu. Il faudrait peutêtre aussi se pencher sur les cas scandinaves et anglais.

Mais si on admet l'hypothèse d'une précocité de l'enseignement de la géographie dans l'espace germanique, cela pourrait être dû à deux facteurs. D'un côté, le morcellement politique ancien du Saint-Empire, réduit mais pas supprimé par les bouleversements de la période révolutionnaire et napoléonienne, avec l'affermissement de territorialités concurrentes, a engendré le besoin d'un enseignement de la géographie aux écoliers pour créer des identités territoriales au service de la légitimation des dynasties prussiennes, bavaroises, saxonnes ..., sans que celles-ci aient réussi à en garder le contrôle, car une entité supérieure allemande apparut dans les enseignements. Ces guerres n'ont pas provoqué l'éveil des masses, en tout cas pas en Allemagne, comme on l'a postulé longtemps, même si certains auteurs le font toujours. L'exemple de l'enseignement de la géographie dans les écoles élémentaires laisse cependant deviner le processus de formation d'une identité territoriale liée à l'État-nation, mais aussi la persistance d'une identité "régionale". En effet, les pédagogues, comme les maîtres d'école, appartenaient bel et bien à l'élite instruite, dont une partie a exalté le sentiment national allemand en 1813 et après. Les nombreuses rééditions de livres de lecture dédiés à un public spécifique comme L'Ami de l'enfant allemand (décrivant amplemement la Prusse natale de son auteur) et L'Ami de l'enfant bavarois pendant les décennies après 1815 indiquent que ces livres ont connu un usage massif, qui a contribué, très lentement, mais progressivement, à donner aux enfants des classes populaires une identité prussienne, bavaroise, saxonne, mais aussi allemande. D'un autre côté, l'absence, si elle se vérifie, de ce genre de livres de l'espace italien, espagnol ou polonais pour cette période charnière, peut être le résultat d'une alphabétisation et d'un degré de scolarisation moindre dans ces territoires. Et il faut bien, du moins selon les conceptions pédagogiques de l'époque, savoir lire et écrire avant de faire de l'éducation civique et de la géographie ... scolaire. 


\section{BIBLIOGRAPHIE}

Aymes J.-R., 1985, « Les « Ilustrados» espagnols face au clergé et à l'enseignement de la religion dans les écoles élémentaires et techniques», Aymes J.-R., Fell E.-M., Guerena J.-L.

(dir.), L'enseignement primaire en Espagne et en Amérique latine du XVIIIe siècle à nos jours, p. 41-64.

Boli J., Ramirez F.,1987, « The Political Construction of Mass Schooling: European Origins and Worldwide Institutionalization», Sociology of Education, 60, pp. 2-17.

Boli J., Ramirez F., Meyer J., 1985, « Explaining the Origins and Expansion of Mass Education »Comparative Education Review, 29(2), pp. 145-170

Böning H., Schmitt H., Siegert R., 2007, Volksaufklärung. Eine praktische Reformbewegung des 18. und 19. Jahrhunderts, Brême, edition lumière, $462 \mathrm{p}$.

Burg van den $\mathrm{M}$, «L'école primaire dans le royaume de Hollande et durant l'annexion à la France ", in Jourdan A. (dir.) Louis Bonaparte: roi de Hollande (1806-1810), Paris, Nouveau Monde, p. 185-197

Cabarrús F, 1808, Cartas sobre los obstáculos que la naturaleza, la opinion y las leyes oponen a la felicidad publica, Vitoria, Pedro Real. $87 \mathrm{p}$.

Clerc P., 2014, « L'enseignement de la géographie : une histoire »

https://www.democratisation-scolaire.fr/spip.php?article193

Czubaty J., 2016, The Duchy of Warsaw, 1807-1815. A Napoleonic Outpost in Central Europe, Londres, Bloomsburry, $258 \mathrm{p}$.

François E., 1989, «Lire et écrire en France et en Allemagne au temps de la Révolution », Berding H., François E., Ullmann, H.-P., (dir.), La Révolution, la France et l'Allemagne. Deux modèles opposés au changement social ? Paris, Maison des Sciences de l'homme, p. 367-384

Furet F., Ozouf J., 1977, Lire et écrire. L'alphabétisation des Français de Calvin à Jules Ferry, Editions de minuit, $392 \mathrm{p}$.

Garner G. 2008, « Statistique, géographie et savoirs sur l'espace en Allemagne (1780-1820) ", Cybergeo revue européenne de géographie [En ligne], Epistémologie, Histoire de la Géographie, Didactique, document 433, mis en ligne le 28 novembre 2008 http://journals.openedition.org/ cybergeo/20883

Gehren K. C., 1816, Karl Christian Gehrens dreimalige Verhaftung und Exportation, Kassel, Marburg, (2e édition), Krieger, $182 \mathrm{p}$.

Gehren K. C., 1810, « Ueber Bildung des Landvolks zum Patriotismus durch Schulunterricht ", Journal für Prediger, vol. 57/I, p. 36-60.

Guermond Y. , 2006, «L'indentité territoriale: l'ambiguïté d'un concept géographique », L'Espace géographique, $\mathrm{n}^{\circ} 4$, p. 292-297.

Haußmann B., 1999, Zwischen Verbauerung und Volksaufklärung. Kurmärkische Landprediger in der zweiten Hälfte des 18. Jahrhunderts, thèse à l'université de Potsdam, Berlin, 258 p.

https://publishup.uni-potsdam.de/opus4-ubp/files/6/haussmann.pdf

Jeismann M., 1992, Das Vaterland der Feinde. Studien zum nationalen Feindbegriff und Selbstverständnis in Deutschland und Frankreich 1792-1918, Stuttgart, Klett-Cotta, 350 p. 
Klewitz, 1805, « Geschichte und Darstellung des Südpreussischen Schulwesens », Neue Berlinische Monatsschrift, Septembre 1805, p. 161-192

Korthals R, 2016, «The education revolution on horseback II: Using the Napoleonic Wars to elicit the effect of tracking on student performance » ROA Research Memoranda, no. 006, Research Centre for Education and the Labour Market.

https://cris.maastrichtuniversity.nl/portal/en/publications/the-education-revolution-onhorseback-ii-using-the-napoleonic-wars-to-elicit-the-effect-of-tracking-on-studentperformance(fd962b85-94a5-4bee-baa7-1061c0f18047)/export.html

Lefort I., 1998, « Deux siècles de géographie scolaire », Espace Temps, p. 146-154

Laboulais-Lesage I., 2001, « Les géographes français de la fin du xviiie siècle et le terrain, recherches sur une paradoxale absence », L'Espace géographique 2001/2 (tome 30), p. 97-110

Lignereux, Aurelien, 2014, « La guerre, ça sert, d'abord, à faire de la géographie : les Français face à l'Europe de Napoléon ", La géographie historique de l'époque napoléonienne, Todorov, Nicola (dir.), Revue de Géographie historique, n 5, 2014/2, http://rgh.univ-lorraine.fr/

Malte-Brun C., 1810, Précis de la géographie universelle, t. I, Paris, Buisson, 548 p.

Marzagalli S., 2016, « Administrer à la française une ville allemande. Les difficultés au quotidien vues de Hambourg », Boudon J.-O., Clemens G., Horn P. dir.), Erbfeinde im Empire ? Franzosen und Deutsche im Zeitalter Napoleons, Thorbecke, p. 97-116,

Mayer J. B., 1816, Der baierische Kinderfreund. Ein Lesebuch für Stadt- und Landsschulen, Munich. Fleischmannschen Buchhandlung, 254 p.

Muyt P. N., 1814, Aardrijksbeschrijving voor Kinderen, ten Gebruike der Schoolen, Leyde, Du Mortier, 49 p.

Neugebauer W., 1985, Absolutistischer Staat und Schulwirklichkeit in Brandenburg-Preussen, Berlin, de Gruyter, 721 p.

Noël J.-F. 2013, « Das Reichsbewusstsein des einfachen Volkes im Deutschland des 18.

Jahrhunderts » Trivium Revue franco-allemande de sciences humaines et sociales14 Le Saint-Empire à l'époque moderne

O'Connor A., 2017, In Pursuit of Politics: Education and Revolution in Eighteenth-century France, Manchester, Manchester University Press, 261 p.

Paye C., 2013, « Almanach royal de Westphalie und Volkskalender im Vergleich. Ein Beitrag zur Kommunikations- und Zensurgeschichte im Königreich Westfalen (1807 - 1813)», Lüsebrink H.-J., Mix, Y.-G., Französische Almanachkultur im deutschen Sprachraum (1700 - 1815), Göttingen, V\&R, p. 227-253.

Péaud, L., 2016, La géographie, émergence d'un champ scientifique. France, Prusse et Grande-Bretagne (1780-1860), Lyon, ENS Éditions, coll. « Sociétés, Espaces, Temps », 280 p.

Planert U., 2007, Der Mythos vom Befreiungskrieg. Frankreichs Kriege und der deutsche Süden: Alltag - Wahrnehmung - Deutung 1792-1841, Paderborn, Schöningh, 739p.

Rochow, F. E. von, 1776, Der Kinderfreund. Ein Lesebuch in zum Gebrauch in Landschulen, Francfort, Eichenbergische Erben, 112 p.

Saugnieux, J., 1985, «Les problèmes de l'alphabétisation dans l'Espagne du XVIIIe siècle », Aymes J.-R, Fell E.-M., Guereña J.-L., (dir.) L'enseignement primaire en Espagne et en Amérique latine du XVIIIe siècle à nos jours, p. 19-29 
Scheerder H.C., 1809, Allereerste beginselen der aardrijkskunde, Harlem, François Bohn, 16 p. Severin-Barboutie B., 2008, Franzosische Herrschaftspolitik und Modernisierung Verwaltungs- und Verfassungsreformen im Großherzogtum Berg (1806-1813) Munich, Oldenbourg, 410 p.

Simien C., 2018, « Des maîtres d'école aux instituteurs : une histoire de communautés rurales, de République et d'éducation, entre Lumières et Révolution (années 1760 - 1802) » Annales historiques de la Révolution française, $\mathrm{n}^{\circ} 2$ p. 189-202

Simsch, A., 1983, Die Wirtschaftspolitik des preussischen Staates in der Provinz Südpreußen 1793-1806/7, Berlin, Duncker \& Humblot, 273 p.

Thieme K. T, 1794, Gutmann oder der Sächsische Kinderfreund. Ein Lesebuch für Bürger- und LandSchulen, Leipzig, Grusius, 268 p.

Todorov N. P., 2011, L'administration du royaume de Westphalie de 1807 à 1813. Le département de l'Elbe, Saarbruck, EUE. 632 p.

Winiarz A., 2016, « Literatura pedagogiczna nauczyciela szkoły elementarnej w KsiĘstwie Warszawskim i Królestwie Polskim (1807-1831) », Prace naukowe Akademii im. Jana Długosza w CZĘstochowie Pedagogika, t. XXV, nr 2, s. 361-374

Zerrenner, C. C., 1811, Der westphälische Kinderfreund, Halle, Kümmel X+ 306 p.

Zerrenner C. C., 1815, Der deutsche Kinderfreund, Halle, Kümmel, 148 p.

Zerrenner C C.,1816, Methodenbuch für Volksschullehrer, Magdeburg, Heinrichshofen, 509 p.

\section{NOTES}

1. Archiwum Główne Akt Dawnych (Archives historiques centrales de Varsovie, par la suite AGAD), GD Südpreussen, $n^{\circ} 1621$, calculé d'après les indications circonstanciées contenues dans les tableaux envoyés par la chambre provinciale à l'administration centrale de Berlin

2. AGAD, GD Südpreussen, $n^{\circ} 1621$, rapport en date du 18 décembre 1795

3. AGAD, Südpreussen, $\mathrm{n}^{\circ} 1601$, Propositions de l'administration provinciale de Varsovie au governement à Berlin, en date du 12 novembre 1799.

4. Ergänzungsblätter zur Allgemeinen Literaturzeitung, n80, juillet 1815, p. 637-638.

\section{RÉSUMÉS}

Les dernières décennies $\mathrm{du} \mathrm{XVIII}^{\mathrm{e}}$ siècle sont caractérisées par un renouvellement des conceptions pédagogiques, qui sont universalistes. La période révolutionnaire et napoléonienne voit entrer la géographie dans le cercle des disciplines enseignées dans les écoles primaires, mais inégalement selon les différents pays d'Europe. Comme la discipline scolaire géographique joue généralement un rôle particulier dans l'affermissement de consciences territoriales, on s'interrogera sur son rôle durant ces décennies charnières dans l'enseignement, mais on cherchera aussi à expliquer les différences géographiques observées à l'aide de l'étude de livres de lecture et de la littérature pédagogique de plusieurs pays d'Europe. 
The last decades of the $18^{\text {th }}$ century saw a renewal of pedagogical thinking, characterized by enlightenment universalism. The Revolutionary and Napoleonic era also saw enter geography primary school teaching as distinct subject, but at different paces in the different parts of Europe. Since the subject "geography" often plays a particular role in forging territorial identities, this article aims at analysing the role of geography in primary schools during this period, but also seeks to explain spatial differences by studying pedagogical litterature printed and disseminated in different European countries.

\section{INDEX}

Mots-clés : enseignement primaire, alphabétisation, géographie scolaire, identités territoriales, livres de lecture

Keywords : primary school teaching, literacy, school geography, territorial identities, reading books

\section{AUTEUR}

\section{NICOLA TODOROV}

Université de Guyane et CRHXIX (EA 3550,PARIS 1 ET 4) 